Short Paper

\title{
Silomais-Sortenprüfung in Südtirol für das Jahr 2019
}

\author{
Variety trials of silage maize in South Tyrol for the year 2019 \\ Prova varietale di mais da trinciato in Alto Adige per l'anno 2019
}

Franziska Mairhofer ${ }^{1}$, Giovanni Peratoner ${ }^{1}$, Matthias Wenter ${ }^{1}$, Ulrich Figl ${ }^{1}$, Aldo Matteazzi ${ }^{1}$

${ }^{1}$ Versuchszentrum Laimburg, Pfatten, Italien

\section{ABSTRACT}

Since silage maize varieties are bred under climatic conditions different from those prevailing in the mountain area of South Tyrol, the silage maize varieties currently available on the market are locally tested every year since 1976. Local variety testing is of great importance because, together with results and information of variety trials from neighboring countries with similar climatic conditions, it allows identifying suitable, adapted varieties under local conditions. A total number of 45 silage maize varieties were tested 2019 in the variety testing program of South Tyrol. Based on the results of these trials, a recommendation list with the best suited varieties for the different South Tyrolean climatic areas is drawn up annually and directly implemented by the few local companies selling maize seed.

\section{KEYWORDS}

silage maize, local variety testing, South Tyrol, variety

recommendation

\section{CITE ARTICLE AS}

Mairhofer Franziska, Peratoner Giovanni, Wenter Matthias et al. (2020). Variety trials of silage maize in South Tyrol for the year 2019. Laimburg Journal 02/2020 DOI: $10.23796 /$ LJ/2020.004

\section{CORRESPONDING AUTHOR}

Franziska Mairhofer

Laimburg 6, Pfatten, I-39040 Auer (BZ), Italien

franziska.mairhofer@laimburg.it $+390471969660$ 


\section{EINLEITUNG}

Die Anbaufläche von Silomais (Zea mays) in Südtirol ist relativ klein und wird auf etwa 1200 ha Ackerfläche geschätzt [1]. Ein breiterer Anbau wird einerseits von den klimatischen Bedingungen des Berggebiets (kurze Vegetationsperiode und Mangel an Wärmeangebot) und andererseits von der Knappheit an ackerfähigen Flächen in den Hanglagen am Berg verhindert, denn in den klimatisch günstigeren Tallagen wird vorwiegend Obst- und Weinbau betrieben. Angesichts des hohen Ertragspotenzials und der hohen Futterqualität [1], des relativ niedrigen Arbeitszeitbedarfs bei leichter Mechanisierung vom Anbau bis hin zur Konservierung sowie der niedrigeren Produktionskosten im Vergleich zu den anderen Formen der Futterproduktion [2] ist Silomais aber für die Landwirte auch eine vorteilhafte und von ihnen ausgesprochen gefragte Kultur. Da Silomais-Sorten meist unter klimatischen Bedingungen gezüchtet werden, die sich von jenen des Berggebiets in Südtirol unterscheiden, werden am Versuchszentrum Laimburg seit 1976 jährlich die aktuell erhältlichen Silomais-Sorten lokal geprüft. Lokale Sortenprüfungen haben eine hohe Wichtigkeit, denn sie ermöglichen, zusammen mit Ergebnissen und Informationen aus Nachbarländern mit ähnlichen klimatischen Verhältnissen ([3] [4] [5] [6] [7]), geeignete, angepasste Sorten für das entsprechende Gebiet zu finden. Aufgrund der Ergebnisse der Sortenprüfung wird jährlich eine Empfehlungsliste mit den besten Sorten für die unterschiedlichen klimatischen Lagen erstellt und von den wenigen Saatgut verkaufenden Firmen direkt umgesetzt.

\section{MATERIAL UND METHODEN}

\section{SORTENWAHL}

In Absprache mit den Züchtungsfirmen werden die Silomais-Sorten für die Sortenprüfung ausgewählt. Grundvoraussetzung ist, dass die Sorten im Firmensortiment herausragende Sorten sind, dass sie klimatisch gut in das Anbaugebiet Südtirol passen und dass sie voraussichtlich über eine längere Zeit am Markt verfügbar sein werden. Letzterer Punkt ist vor allem deshalb ausschlaggebend, da die Silomais-Sorten mindestens zwei Jahre, meist aber drei Jahre geprüft werden, bevor sie bei entsprechenden Ergebnissen in die Sorten-Empfehlungsliste aufgenommen werden.
Auch jene Silomais-Sorten, welche schon in die Südtiroler Sorten-Empfehlungsliste aufgenommen wurden, werden immer wieder getestet, solange sie in der Empfehlungsliste bleiben. Damit wird überprüft, ob die Performance der bereits empfohlenen Sorten noch mit den neuen Sorten in der Sortenprüfung mithalten kann.

Weiteres Grundkriterium für die Aufnahme der Sorten ins Prüfsortiment ist die gesetzlich festgelegte, EU-weit geltende GVO-Freiheit, d.h. die Firmen müssen deklarieren, dass die Sorten frei von gentechnisch veränderten Organismen sind.

Die Silomais-Sorten werden je nach FAOZahl [8] in vier verschiedene Reifegruppen eingeteilt und an vier klimatisch unterschiedlichen Standorten angebaut. Die Einteilung erfolgt nach den FAO-Zahlen, die die jeweiligen Saatgutfirmen für ihre Sorten angeben. Frühe Silomais-Sorten mit FAO-Zahlen zwischen 190 und 220 werden in höheren Lagen auf etwa $1100 \mathrm{~m}$ ü. NN im Pustertal (Standort Olang), mittelfrühe Sorten mit FAO-Zahlen zwischen 220 und 250 an einem Standort auf etwa $900 \mathrm{~m}$ ü. NN im oberen Vinschgau (Standort Lichtenberg), mittelspäte Sorten mit FAO-Zahlen zwischen 250 und 280 an einem klimatisch günstigen Standort bei etwa $900 \mathrm{~m}$ ü. NN im Pustertal (Standort Dietenheim) und späte Sorten mit FAO-Zahlen von 300 bis 500 an einer klimatisch günstigen Lage auf etwa $740 \mathrm{~m}$ ü. NN im Eisacktal (Standort Gufidaun) getestet (Tab. 1).

\section{VERSUCHSDESIGN}

2019 wurden insgesamt 45 Silomais-Sorten geprüft: 10 Sorten in Olang (FAO-Zahlen von 190 bis 220), 9 Sorten in Lichtenberg (FAOZahlen von 220 bis 250), 18 Sorten in Dietenheim (FAO-Zahlen von 250 bis 280) und 12 Sorten in Gufidaun (FAO-Zahlen von 300 bis 500) (Tab. 2). Mit Ausnahme der Populationssorte Evolino handelte es sich bei allen anderen getesteten Sorten um Hybridsorten.

Als Versuchsdesign wurden randomisierte unvollständige Blöcke (Alpha-Gitter) mit drei vollständigen Wiederholungen genutzt. In Olang wurde ein Alpha-Gitter $5 \times 2 \times 3$, in Lichtenberg ein $3 \times 3 \times 3$, in Dietenheim ein $6 \times 3 \times 3$ und in Gufidaun ein $6 \times 2 \times 3$ verwendet. Die Parzellen hatten in Olang, Dietenheim und Lichtenberg eine Größe von je 3,0 $\mathrm{m} x$ $4,5 \mathrm{~m}$ sowie in Gufidaun von je $2,8 \mathrm{~m} \times$ $4,5 \mathrm{~m}$.

\section{ANBAU}

Je nach Standort wurde im Zeitraum von Ende April bis Anfang Mai gesät und von Ende September bis Anfang Oktober geerntet (Tab. 3).

Mit einem Setzstock wurden die Samen entlang von Setzlatten mit Markierungen bei den vorgesehenen Abständen einzeln händisch auf eine Tiefe von $5 \mathrm{~cm}$ abgelegt. Pro Parzelle wurden vier Reihen gesät. Um Randeffekte zu vermindern wurden in Dietenheim und Lichtenberg bei den Randparzellen sechs Reihen gesät, während in Olang und Gufidaun beim Anbau von Silomais um den Versuch der Abstand zur letzten Reihe links und rechts genau eingehalten wurde. Die Unkrautbekämpfung erfolgte im frühen Nachauflauf in Dietenheim und Gufidaun mit den Wirkstoffen S-Metolachlor, Terbuthylazine und Mesotrione und in Lichtenberg mit den Wirkstoffen S-Metolachlor, Terbuthylazine und Mesotrione sowie Dicamba. Am Standort Olang erfolgte eine Behandlung im frühen Nachauflauf mit den Wirkstoffen Thiencarbazon-methyl, Cyprosulfamide und Isoxaflutole sowie eine zweite Behandlung im Nachauflauf mit Dicamba (Tab. 3).

Die Aussaat fand 2019 zwei bis sechs Tage später als 2018 statt. Durch die kühlen Temperaturen im Mai (Tab. 4) verzögerten sich das Auflaufen und die Jugendentwicklung der Pflanzen deutlich. 2019 waren mit 23,4\% im Vergleich zum mehrjährigen Mittel (2005-2018) von 3,0\% deutlich mehr Nebentriebe vorhanden, was an den kühlen Temperaturen im Mai beim Auflaufen und bei der Jugendentwicklung liegen könnte.

Durch den überdurchschnittlich warmen Juni konnte sich der Mais aber rasch erholen und die Wachstumsverzögerung wettmachen. Die Siloreife (Teigreife) wurde an allen vier Standorten später als im Vorjahr erreicht, sodass erst ab der vierten Septemberwoche vier bis dreizehn Tage später als 2018 geerntet werden konnte.

Die Ernte erfolgte bei Erreichung der Siloreife (Teigreife). Im Normalfall wurden die zwei mittleren der vier Reihen jeweils als eigenständige Probe geerntet. Wiesen diese Reihen besondere Anomalien wie erhöhten Vogelfraß oder ungewöhnlich viele verkümmerte Pflanzen auf, wurde auf eine der beiden Außenreihen ausgewichen. Zunächst erfolgte eine Bonitur der Reihen im Bestand. Es wurden die Sollpflanzen in der Reihe gezählt sowie Fehlstellen, Schwachpflanzen 
und Nebentriebe erfasst. Nach der Bonitur wurde jede Reihe mittels Sichel bei einer Schnitthöhe von $15 \mathrm{~cm}$ händisch geschnitten und das Frischgewicht mittels einer Paketwaage mit XL-Plattform (EOS 150K5OXL, KERN \& SOHN GmbH, Balingen, Deutschland) bestimmt. Im Anschluss wurde jede Probe mit Hilfe des traktorgetragenen Maishäckslers gehäckselt. Das Häckselgut einer ganzen Reihe wurde in einer Wanne aufgefangen und gemischt. Eine Probe mit 500 g Frischgewicht wurde entnommen und umgehend zum Labor transportiert.

\section{LABORAUSWERTUNG}

Die Proben wurden im Trockenschrank bei $60{ }^{\circ} \mathrm{C}$ bis zur Gewichtskonstanz getrocknet. Im Anschluss wurde das Trockengewicht gemessen und die Proben mittels Universal-Schneidmühle mit Hochleistungszyklon (Pulverisette 25 mit Hochleistungszyklon 19, Fritsch $\mathrm{GmbH}$, IdarOberstei, Deutschland) durch ein $0,75 \mathrm{~mm}$-Sieb vermahlen. Die Proben wurden mittels Nahinfrarot-Spektrometer ohne Standardisierung (NIRSsystem 5000, FOSS A/S, Hillerød, Denmark) gescannt. Die erhaltenen Spektren wurden über Kalibrationskurven (sm2019-1.eqa) der VDLUFA Qualitätssicherung NIRS (Verband Deutscher Landwirtschaftlicher Untersuchungs- und Forschungsanstalten, Darmstadt, Deutschland) ausgewertet und Stärkegehalt, Energiegehalt, Rohprotein und Rohfaser bestimmt. Aufgrund der Anzahl an Pflanzstellen pro Reihe wurde die Referenzfläche bestimmt und ausgehend von den Labordaten der TM-Ertrag und der Energieertrag rechnerisch ermittelt.

\section{STATISTISCHE AUSWERTUNG}

Die Ergebnisse wurden getrennt für jeden Standort mittels GLM (General Linear Model) ausgewertet. Die Sorte wurde als fixer Effekt, die Wiederholung und ihre Wechselwirkung mit dem Block als zufällige Effekte berücksichtigt. Post-hoc-Vergleiche der geschätzten Randmittelwerte der Sorten erfolgten nach LSD (Least Significant Difference).

Das Vorhandensein der Voraussetzungen (Normalverteilung der Residuen, Varianzhomogenität) wurde nach Inspektion der Residuen durchgeführt. Bei Verletzung der Voraussetzungen wurde die abhängige Variable transformiert. Rücktransformierte Randmittelwerte werden für diese

\section{ZUSAMMENFASSUNG}

Da die auf dem Markt erhältlichen Silomais-Sorten unter anderen klimatischen Bedingungen gezüchtet werden als sie im Berggebiet Südtirols vorherrschen, werden seit 1976 jährlich Silomais-Sorten lokal geprüft. Lokale Sortenprüfungen sind von großer Bedeutung, denn sie ermöglichen, zusammen mit den Ergebnissen und Informationen der Sortenversuche aus Nachbarländern mit ähnlichen klimatischen Verhältnissen, geeignete, angepasste Sorten für das entsprechende Gebiet zu finden. 2019 wurden bei der Silomais-Sortenprüfung in Südtirol insgesamt 45 SilomaisSorten getestet. Aufgrund der Ergebnisse dieser Sortenprüfung wird jährlich eine Empfehlungsliste mit den am besten geeigneten Sorten für die unterschiedlichen Lagen Südtirols erstellt und von den wenigen Saatgut verkaufenden Firmen direkt umgesetzt.

\section{RIASSUNTO}

Poiché le varietà di mais da trinciato disponibili sul mercato vengono allevate in condizioni climatiche diverse da quelle prevalenti nella zona montana dell'Alto Adige, dal 1976 le varietà di mais da trinciato vengono testate ogni anno a livello locale. I test varietali locali sono di grande importanza perché, insieme ai risultati e alle informazioni dei test varietali dei paesi vicini con condizioni climatiche simili, permettono di trovare varietà adatte e adattate alla rispettiva zona. Nel programma di test varietali dell'Alto Adige sono state testate nel 2019 un totale di 45 varietà di mais da trinciato. Sulla base dei risultati di questi test varietali, ogni anno viene stilato un elenco di raccomandazioni con le varietà più adatte alle diverse zone climatiche dell'Alto Adige, che viene attuato direttamente dalle poche aziende locali che vendono sementi di mais.

Variablen gezeigt. Das Signifikanzniveau wurde auf $5 \%$ festgesetzt.

Alle statistischen Auswertungen erfolgten mit IBM SPSS Statistics 24 (IBM, Armonk, New York, USA).

\section{ERGEBNISSE UND DISKUSSION}

Die Ergebnisse der Silomais-Sortenprüfung für die vier Standorte sind in Tabelle 5, Tabelle 6, Tabelle 7 und Tabelle 8 dargestellt.

Mit wenigen Ausnahmen erreichten alle Sorten der vier Standorte eine gute Qualität mit einem Stärkegehalt um 35-36\% und einem Energiegehalt von mehr als 6,5 MJ NEL/kg Trockensubstanz. Insbesondere an den Standorten Gufidaun und Lichtenberg war der durchschnittliche Energiegehalt aller Sorten mit Werten von 7,55 bzw. 7,67 MJ NEL/kg Trockensubstanz deutlich überdurchschnittlich.

Die Trockenmasseerträge waren im mehrjährigen Vergleich in Dietenheim mit
28,4 t/ha (Durchschnitt 1977-2019: $23,7 \mathrm{t} / \mathrm{ha}$ ), in Olang mit 23,4 t/ha (Durchschnitt 2008-2019 (ohne 2017): 21,6 t/ha) und Gufidaun mit 29,6 t/ha (Durchschnitt 2011-2019: 28,8 t/ha) deutlich überdurchschnittlich. Nur in Lichtenberg lagen die Trockenmasseerträge mit 23,2 t/ha (Durchschnitt 2003-2019: 23,8t/ha) etwas unter dem mehrjährigen Durchschnitt.

Die späte Sorte P0729 (Pioneer) und die frühe Sorte $P 7500$ (Pioneer) wurden aufgrund der mehrjährigen überdurchschnittlichen Ergebnisse in die Sorten-Empfehlungsliste 2020 (www.laimburg.it/de/dienstleistungen/anleitungen.asp?somepubl cate id=20206\&news action=4\&news article id=634635) neu aufgenommen. Die späte Sorte P0729 (FAO-Zahl 400) war über die drei Prüfjahre hinweg insbesondere bei Trockenmasseertrag ( $\mathrm{t} / \mathrm{ha}$ ) und Energieertrag (GJ NEL/ha), aber auch bei Stärkegehalt (\%) und Energiegehalt (MJ NEL/kg) überlegen (Abb. 1) (Tab. 9). Auch bei den KörnermaisSortenprüfungen in Italien zählt die Sorte 
P0729 zu den produktivsten Hybridsorten mit der FAO-Zahl 400: Der Produktionsindex der Sorte (die relativen Werte beziehen sich auf den Mittelwert des Sortiments im jeweiligen Prüfjahr) war in den letzten vier Jahren stets deutlich überdurchschnittlich [9]. Die frühe Sorte P7500 (FAO-Zahl 210) zeigte trotz einiger Schwankungen über die fünf Prüfjahre hinweg vor allem bei Stärkegehalt (\%) und Energiegehalt (MJ NEL/kg) überdurchschnittliche Werte bei nahezu durchschnittlichem TM-Ertrag (Abb. 2).

\section{LITERATUR}

[1] Peratoner G., Kasal A., Plitzner C. (2010). Stima del bilancio foraggero per l'Alto Adige. In: Bovolenta S. (ed.): Zootecnica e montagna: Quali strategie per il futuro? Quaderno SoZooAlp 6. Nuove Arti Grafiche, Trento, Italia, pp. 111-122. Retrieved March 16, 2020, from https://www.sozooalp.it/fileadmin/superuser/Quaderni/quaderno 6/8 Peratoner SZA6.pdf.

[2] Peratoner G., Figl U., Florian C. et al. (2015). Studio dei costi di produzione del foraggio nella Provincia di Bolzano (BLW-gw-11-1). Relazione finale di progetto. Versuchszentrum Laimburg, Vadena/Pfatten, Italien.

[3] Österreichische Agentur für Gesundheit und Ernährungssicherheit GmbH - AGES (ed.) (2020). Österreichische Beschreibende Sortenliste 2020 (Auszug). Landwirtschaftliche Pflanzenarten. Republik Österreich. Gemäß Saatgutgesetz 1997. (Schriftenreihe ; 10/2020). Retrieved March 5, 2020, from https://bsl.baes.gv.at/filead$\mathrm{min} / \mathrm{BSL} / \mathrm{pdfVer}$ sion/Auszug 2020 gesamt.pdf.
Die Sorte $P 7500$ wurde in Bayern in einer Grenzlage für frühe Sorten angebaut und zeigte dort aber durchwegs unterdurchschnittliche Werte [10].

\section{DANKSAGUNGEN}

Ein besonderer Dank gilt den Landwirten Peter und Thomas Dorfmann aus Gufidaun, Anton Wallnöfer aus Lichtenberg und Gebhard, Martin und Matthias Monthaler aus Olang sowie Michael Monthaler von der
Agentur Landesdomäne. Dem Hydrografischen Amt in Bozen gilt ein Dank für die Bereitstellung der meteorologischen Daten. Dank gilt auch allen, die bei Aussaat und Ernte des Silomais mitgeholfen haben: Mattia Bonet, Sabine Franzelin, Michael Gasser, Egon Hilber, Thomas Holtz, Alexander Kofler, Paul Mittermair, Maximilian Morlacchi, Helmut Oberleiter, Manuel Pramsohler, Martina Querini, Reinhold Seiwald, Patrick Tinney, Barbara Waldboth und Bastian Zwack.
[4] Hiltbrunner J., Buchmann U., Pignon P. et al. (2020). Liste der empfohlenen Maissorten für die Ernte 2020. Agroscope Transfer 314, 1-6.

[5] Landwirtschaftliches Zentrum Baden-Württemberg LAZBW (2020). Aktuelle Sortenergebnisse der LSV Silomais. Retrieved March 16, 2020, https://lazbw.landwirtschaftbw.de/pb/,Lde/Startseite/Themen/Aktuelle+Sortenergebnisse+der+LSV+Mais.

[6] Bayerische Landesanstalt für Landwirtschaft (LfL) Pflanzenbau (2020a). Sortenbeschreibung Mais 2019/2020. Retrieved March 5, 2020, from https://www.Ifl.bayern.de/mam/cms07/ipz/dateien/sm19 sortenbeschreibung.pdf.

[7] Bayerische Landesanstalt für Landwirtschaft (LfL) Pflanzenbau (2020b). Regionale Sortenberatung in Bayern 2019/2020. Empfehlungssorten Silomais. Retrieved March 05, 2020, from https://www.Ifl.bayern.de/mam/cms07/ipz/dateien/sm19 empfehlungssorten 2020.pdf.
[8] Deutsches Maiskomitee e.V. (2020). Bestimmung Reifezahl. Retrieved March 09, 2020, from https://www.maiskomitee.de/Produktion/Sorten/Bestimmung-Reifezahl.

[9] Andreotti, L. (2020). Ibridi di mais per le semine 2020. L'Informatore Agrario 76 (3), 27 50.

[10] Bayerische Landesanstalt für Landwirtschaft (LfL) Pflanzenbau (2020). Ergebnisse der Sortenversuche mit Silomais für Grenzlagen an den Versuchsorten in Bayern 2019. Versuche 301 - Grenzlagen in Nord-Ost Bayern. Ergebnisse: Hartenhof / Neumarkt. Retrieved March 16, from https://www.Ifl.bayern.de/mam/cms07/ipz/dateien/sm19 301 grenzlagen hartenhof.pdf 


\section{ANHANG 1: TABELLEN}

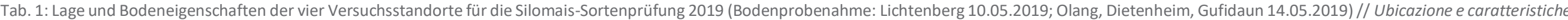
del terreno dei quattro siti sperimentali delle prove varietali di mais da trinciato del 2019 (campionamento del suolo: Montechiaro 10.05.2019; Valdaora, Teodone e Gudon 14.05.2019).

\begin{tabular}{|c|c|c|c|c|c|c|c|c|c|c|c|c|c|c|}
\hline \multirow[t]{2}{*}{$\begin{array}{l}\text { Standort } \\
\text { Sito sperimen- } \\
\text { tale }\end{array}$} & \multirow[t]{2}{*}{$\begin{array}{l}\text { Längengrad } \\
\text { Longitudine } \\
\text { (E) }\end{array}$} & \multirow[t]{2}{*}{$\begin{array}{l}\text { Breitengrad } \\
\text { Latitudine } \\
\text { (N) }\end{array}$} & \multirow[t]{2}{*}{$\begin{array}{l}\begin{array}{l}\text { Meeres- } \\
\text { höhe }\end{array} \\
\text { Altitudine } \\
\text { (m ü. NN) }\end{array}$} & \multirow[t]{2}{*}{$\begin{array}{c}\text { Bodenart } \\
\text { (Oberboden) } \\
\text { Tessitura del } \\
\text { suolo (topsoil) }\end{array}$} & \multicolumn{2}{|c|}{ pH } & \multicolumn{2}{|c|}{$\begin{array}{l}\text { Org. C (als Humus) } \\
\text { Carbonio organico } \\
\text { espresso come humus } \\
\text { (\%) }\end{array}$} & \multicolumn{2}{|c|}{$\begin{array}{c}N_{\min } \\
(\mathrm{kg} / \mathrm{ha})\end{array}$} & \multicolumn{2}{|c|}{$\begin{array}{c}\mathrm{P}_{2} \mathrm{O}_{5} \\
(\mathrm{mg} / 100 \mathrm{~g})\end{array}$} & \multicolumn{2}{|c|}{$\begin{array}{c}\mathrm{K}_{2} \mathrm{O} \\
(\mathrm{mg} / 100 \mathrm{~g})\end{array}$} \\
\hline & & & & & $0-30 \mathrm{~cm}$ & $30-60 \mathrm{~cm}$ & $0-30 \mathrm{~cm}$ & $30-60 \mathrm{~cm}$ & $0-30 \mathrm{~cm}$ & $30-60 \mathrm{~cm}$ & $0-30 \mathrm{~cm}$ & $30-60 \mathrm{~cm}$ & $0-30 \mathrm{~cm}$ & $30-60 \mathrm{~cm}$ \\
\hline Olang & $12^{\circ} 02^{\prime} 25,4^{\prime \prime}$ & $46^{\circ} 45^{\prime} 41,9^{\prime \prime}$ & 1.077 & $\begin{array}{l}\text { Humoser toni- } \\
\text { ger Schluff }\end{array}$ & 6,0 & 5,8 & 4,2 & 2,3 & 38,0 & 19,0 & 10,0 & $<3,0$ & 18,0 & $<3,0$ \\
\hline Lichtenberg & $10^{\circ} 34^{\prime} 45,34^{\prime \prime}$ & $46^{\circ} 38^{\prime} 15,76^{\prime \prime}$ & 890 & $\begin{array}{l}\text { Humoser sandi- } \\
\text { ger Schluff }\end{array}$ & 7,7 & 7,7 & 5,0 & 1,6 & 80,0 & 35,0 & 42,0 & 19,0 & 80,0 & 42,0 \\
\hline Dietenheim & $11^{\circ} 57^{\prime} 50,17^{\prime \prime}$ & $46^{\circ} 47^{\prime} 48,74^{\prime \prime}$ & 920 & Lehmiger Sand & 5,8 & 6,0 & 3,8 & 2,2 & 181,0 & 50,0 & 24,0 & 6,0 & 44,0 & 15,0 \\
\hline Gufidaun & $11^{\circ} 35^{\prime} 53,03^{\prime \prime}$ & $46^{\circ} 38^{\prime} 51,46^{\prime \prime}$ & 743 & $\begin{array}{l}\text { Stark humoser } \\
\text { lehmiger Sand }\end{array}$ & 7,1 & 7,1 & 8,2 & 3,5 & 171,0 & 130,0 & 170,0 & 100,0 & 135,0 & 94,0 \\
\hline
\end{tabular}


Tab. 2: Angebaute Sorten bei der Silomais-Sortenprüfung 2019 // Varietà testate nelle prove varietali del 2019.

\begin{tabular}{|c|c|c|c|}
\hline $\begin{array}{l}\text { Sortenname } \\
\text { Nome varietà }\end{array}$ & $\begin{array}{c}\text { Bezugsfirma } \\
\text { Ditta sementiera }\end{array}$ & $\begin{array}{l}\text { FAO-Zahl* } \\
\text { Classe FAO* }\end{array}$ & $\begin{array}{l}\text { Prüfstandorte** } \\
\text { Siti sperimentali** }\end{array}$ \\
\hline Adevey & Limagrain & 280 & D \\
\hline ATRANS & Saatzucht Gleisdorf & 290 & D \\
\hline Belami & Caussade Semences & 190 & $\mathrm{O}$ \\
\hline Benedictio & KWS & 230 & L \\
\hline Evolino & gzpk & 220 & L \\
\hline Fabregas & KWS & 210 & $\mathrm{O}$ \\
\hline Figaro & KWS & 250 & $D, L$ \\
\hline Frederico & KWS & 240 & L \\
\hline FREEMAN & MAS Seeds & 400 & G \\
\hline Friendli & Caussade Semences & 210 & 0 \\
\hline GIULIANA & Saatzucht Gleisdorf & 280 & D \\
\hline Gottardo & KWS & 220 & L, O \\
\hline Hulk & Agasaat & 250 & D \\
\hline Kentos & KWS & 280 & D \\
\hline Keops & KWS & 210 & 0 \\
\hline KOKUNA & Saatzucht Gleisdorf & 290 & D \\
\hline LG30315 & Limagrain & 270 & D \\
\hline MAS 16.B & MAS Seeds & 220 & 0 \\
\hline MAS 26.R & MAS Seeds & 300 & 0 \\
\hline MELONGA & Saatzucht Gleisdorf & 270 & D \\
\hline P0362 & Pioneer & 400 & G \\
\hline P0729 & Pioneer & 400 & G \\
\hline P0837 & Pioneer & 400 & G \\
\hline P1275 & Pioneer & 500 & G \\
\hline P7378 & Pioneer & 200 & 0 \\
\hline P7500 & Pioneer & 210 & 0 \\
\hline P7515 & Pioneer & 220 & L, O \\
\hline P7932 & Pioneer & 250 & $\mathrm{D}$ \\
\hline P8000 & Pioneer & 230 & L \\
\hline P8201 & Pioneer & 240 & L \\
\hline P8333 & Pioneer & 260 & D \\
\hline P8500 & Pioneer & 260 & D \\
\hline P8666 & Pioneer & 270 & D \\
\hline P8707 & Pioneer & 270 & D \\
\hline
\end{tabular}




\begin{tabular}{lccc} 
P8888 & Pioneer & 280 & D \\
P9911 & Pioneer & 300 & G \\
\hline Poesi & Caussade Semences & 280 & D \\
SIV4795 & Sivam & 300 & G \\
SIV5031 & Sivam & 300 & G \\
SIV6181 & Sivam & 500 & G \\
\hline SY Carioca & Syngenta & 400 & G \\
\hline SY Senko & Syngenta & 400 & G \\
SY Zephir & Syngenta & 300 & G \\
Torres & KWS & 250 & D, L \\
Walterinio & KWS & 270 & D
\end{tabular}

*FAO-Zahl nach deutschem Maiskomitee // Classe FAO secondo il Deutsches Maiskomitee.

** $\mathrm{O}=$ Olang, $\mathrm{L}=$ Lichtenberg, $\mathrm{D}=$ Dietenheim, $\mathrm{G}=$ Gufidaun $/ /{ }^{* *} \mathrm{O}=$ Valdaora, $\mathrm{L}=$ Monte Chiaro, $\mathrm{D}=$ Teodone, $\mathrm{G}=$ Gudon. 
Tab. 3: Anbaudaten für die vier Versuchsstandorte der Silomais-Sortenprüfung 2019 // Conduzione agronomica dei quattro siti sperimentali delle prove varietali del 2019.

\begin{tabular}{|c|c|c|c|c|c|c|c|c|c|}
\hline $\begin{array}{l}\text { Standort } \\
\text { Sito sperimen- } \\
\text { tale }\end{array}$ & Data di semina & $\begin{array}{l}\text { Data di rac- } \\
\text { colta }\end{array}$ & $\begin{array}{l}\text { Precessione col- } \\
\text { turale }\end{array}$ & $\begin{array}{l}\text { Sesto d'impianto } \\
(\mathrm{cm})\end{array}$ & $\begin{array}{c}\text { Pflanzendichte } \\
\text { (Pflanzen } / \mathrm{m}^{2} \text { ) } \\
\text { Densitò } \\
\text { (piante } / \mathrm{m}^{2} \text { ) }\end{array}$ & $\begin{array}{l}\text { Concimazione di } \\
\text { fondo }\end{array}$ & $\begin{array}{l}\text { Kopfdüngung } \\
\text { Concimazione in } \\
\text { copertura }\end{array}$ & Irrigazione & Lotta alle malerbe \\
\hline Olang & 08.05.2019 & 08.10 .2019 & $\begin{array}{l}\text { Mais, Roggen } \\
\text { als Winter-zwi- } \\
\text { schenfrucht }\end{array}$ & $75 \times 12,5$ & 10,6 & $\begin{array}{l}\text { Frühjahr + Herbst je- } \\
\text { weils } 40 \mathrm{~m}^{3} \text { Gülle/ha }\end{array}$ & keine & ja & $\begin{array}{c}\text { 18.05.2019: } 2 \text { I/ha } \\
\text { Thiencarbazon-me- } \\
\text { thyl, Cyprosulfamide } \\
\text { und Isoxaflutole } \\
\begin{array}{c}\text { 25.06.2019: } 1 \text { I/ha Di- } \\
\text { camba }\end{array}\end{array}$ \\
\hline Lichtenberg & 10.05 .2019 & 01.10.2019 & Mais & $75 \times 12,5$ & 10,6 & $\begin{array}{c}\text { Frühjahr: } 50 \mathrm{~m}^{3} \text { Stall- } \\
\text { mist/ha }+44 \mathrm{~m}^{3} \\
\text { Gülle/ha }\end{array}$ & keine & ja & $\begin{array}{c}\text { 27.05.2019: } 4 \text { I/ha S- } \\
\text { Metolachlor, Ter- } \\
\text { buthylazine und Meso- } \\
\text { trione + 0,5 I/ha Di- } \\
\text { camba }\end{array}$ \\
\hline Dietenheim & 02.05 .2019 & 24.09.2019 & Mais & $75 \times 14$ & 9,5 & $\begin{array}{l}\text { Herbst: } 30 \mathrm{~m}^{3} \text { Biogas- } \\
\text { gülle/ha + Frühjahr: } \\
70 \mathrm{~m}^{3} \text { Biogasgülle/ha }\end{array}$ & keine & ja & $\begin{array}{l}\text { 17.05.2019: } 4 \mathrm{l} / \mathrm{ha} \text { S- } \\
\text { Metolachlor, Ter- } \\
\text { buthylazine und Me- } \\
\text { sotrione }\end{array}$ \\
\hline Gufidaun & 30.04 .2019 & 26.09.2019 & Mais & $70 \times 16$ & 8,9 & $\begin{array}{c}\text { Frühjahr: } 20 \mathrm{~m}^{3} \\
\text { Gülle/ha + Mist } \\
\text { (Menge unbekannt) }\end{array}$ & keine & ja & $\begin{array}{l}\text { 07.05.2019: } 4 \mathrm{l} / \mathrm{ha} \text { S- } \\
\text { Metolachlor, Ter- } \\
\text { buthylazine und Me- } \\
\text { sotrione }\end{array}$ \\
\hline
\end{tabular}


Tab. 4: Wetterverlauf an den vier Versuchsstandorten der Silomais-Sortenprüfung 2019 für die Vegetationsperiode (Mai - September) // Andamento meteorologico dei quattro siti sperimentali nel 2019 durante il periodo vegetativo (maggio - settembre).

\begin{tabular}{|c|c|c|c|c|c|}
\hline \multirow[t]{2}{*}{ Standort } & \multirow[t]{2}{*}{ Stazione meteorologica } & \multicolumn{2}{|l|}{$\begin{array}{l}\text { Temperaturmittelwert } \\
\text { Mai-September } \\
\text { Media maggio-settembre } \\
\left({ }^{\circ} \mathrm{C}\right)\end{array}$} & \multicolumn{2}{|c|}{ Niederschlag Mai-September } \\
\hline & & $\begin{array}{l}\text { Langjähriges Mittel } \\
\text { (1997-2019) } \\
\text { Media di lungo periodo } \\
\text { (1997-2019) }\end{array}$ & 2019 & $\begin{array}{l}\text { Langjähriges Mittel } \\
\text { (1997-2019) } \\
\text { Media di lungo periodo } \\
\text { (1997-2019) }\end{array}$ & 2019 \\
\hline Gufidaun & $\begin{array}{l}\text { Klausen-Säben } \\
\text { (700 m ü. NN) }\end{array}$ & 18,0 & 18,4 & 462 & 393 \\
\hline Lichtenberg & $\begin{array}{c}\text { Eyrs } \\
\text { (874 m ü. NN) }\end{array}$ & 15,9 & 15,8 & 311 & 339 \\
\hline Dietenheim & $\begin{array}{l}\text { Dietenheim } \\
\text { (850 m ü. NN) }\end{array}$ & 15,9 & 15,7 & 550 & 435 \\
\hline Olang & $\begin{array}{l}\text { Stausee Olang } \\
\text { (1057 m ü. NN) }\end{array}$ & 14,3 & 15,4 & 486 & 369 \\
\hline
\end{tabular}


Tab. 5: Ergebnisse der Silomais-Sortenprüfung 2019 für den Standort Olang. // Risultati delle prove varietali 2019 a Valdaora.

\begin{tabular}{|c|c|c|c|c|c|c|c|c|c|}
\hline \multirow{3}{*}{$\begin{array}{l}\text { Sorte (FAO-Zahl) } \\
\text { Varietà e classe } \\
\text { FAO }\end{array}$} & \multirow{3}{*}{$\begin{array}{l}\text { TS-Gehalt } \\
\text { Sostanza } \\
\text { secca } \\
(\%)\end{array}$} & \multicolumn{2}{|c|}{ TM-Ertrag } & \multirow{3}{*}{$\begin{array}{l}\text { Stärkegehalt } \\
\text { Amido } \\
\text { (\%) }\end{array}$} & \multirow{3}{*}{$\begin{array}{l}\text { Energiegehalt } \\
\text { Concentrazione } \\
\text { energetica } \\
\text { (MJ NEL/kg TS) }\end{array}$} & \multirow{2}{*}{\multicolumn{2}{|c|}{$\begin{array}{l}\text { Energieertrag } \\
\text { Resa energetica }\end{array}$}} & \multirow{3}{*}{$\begin{array}{l}\text { Fehlstellenw } \\
\text { Fallanze }^{w} \\
(\%)\end{array}$} & \multirow{3}{*}{$\begin{array}{l}\text { Nebentriebe } \\
\text { II e III piante } \\
\text { (\%) }\end{array}$} \\
\hline & & \multicolumn{2}{|c|}{ Produzione sostanza secca } & & & & & & \\
\hline & & (t/ha) & (rel. \%) & & & (GJ NEL/ha) & (rel. \%) & & \\
\hline Belami (190) & $38,5^{\mathrm{bc}}$ & 22,7 & 96,9 & $35,5^{a b}$ & $6,98^{a}$ & 158,6 & 98,6 & $17,6^{\mathrm{cd}}$ & $12,2^{\mathrm{cd}}$ \\
\hline Fabregas (210) & $40,0^{b}$ & 22,6 & 96,5 & $34,7^{b}$ & $6,83^{\mathrm{bc}}$ & 154,5 & 96,0 & $20,3^{b c d}$ & $25,3^{\mathrm{ab}}$ \\
\hline Friendli (210) & $36,1^{c d}$ & 24,4 & 104,1 & $35,8^{b}$ & $6,88^{a b c}$ & 168,8 & 104,9 & $20,6^{b c d}$ & $5,3^{d}$ \\
\hline Gottardo (220) & $39,4^{b}$ & 22,2 & 94,8 & $39,8^{a}$ & $6,94^{\mathrm{ab}}$ & 154,9 & 96,3 & $22,7^{b c}$ & $17,0^{\mathrm{bcd}}$ \\
\hline Keops (210) & $35,5^{d}$ & 21,6 & 92,2 & $34,9^{b}$ & $6,94^{\mathrm{ab}}$ & 150,0 & 93,2 & $28,3^{a}$ & $15,2^{\mathrm{cd}}$ \\
\hline MAS 16.B (220) & $35,0^{d}$ & 25,2 & 107,6 & $34,7^{b c}$ & $6,90^{\mathrm{abc}}$ & 174,2 & 108,3 & $17,5^{d}$ & $21,6^{b c}$ \\
\hline MAS 26.R (300) & $28,0^{e}$ & 23,5 & 100,3 & $30,4^{c}$ & $6,53^{e}$ & 154,4 & 96,0 & $22,2^{\mathrm{bcd}}$ & $21,7^{b c}$ \\
\hline P7378 (200) & $42,7^{a}$ & 24,1 & 102,9 & $38,0^{\mathrm{ab}}$ & $6,68^{d}$ & 161,6 & 100,4 & $24,3^{\mathrm{ab}}$ & $31,8^{a}$ \\
\hline P7500 (210) & $39,4^{b}$ & 24,5 & 104,6 & $38,3^{\mathrm{ab}}$ & $6,96^{\mathrm{ab}}$ & 171,8 & 106,8 & $23,2^{b}$ & $14,6^{\mathrm{cd}}$ \\
\hline P7515 (220) & $37,3^{\mathrm{bcd}}$ & 23,5 & 100,3 & $38,0^{\mathrm{ab}}$ & $6,78^{\mathrm{cd}}$ & 160,1 & 99,5 & $20,6^{b c d}$ & $19,9^{b c}$ \\
\hline $\begin{array}{l}\text { Mittelwert } \\
\text { Media }\end{array}$ & 37,2 & 23,4 & & 36,0 & 6,84 & 160,9 & & 21,7 & 18,5 \\
\hline
\end{tabular}

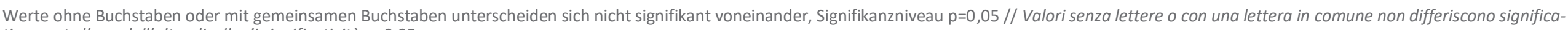
tivamente l'uno dall'altro, livello di significatività $p=0,05$.

W Auswertung nach Winkeltransformation // W Elaborazione dopo trasformazione angolare. 
Tab. 6: Ergebnisse der Silomais-Sortenprüfung 2019 für den Standort Lichtenberg // Risultati delle prove varietali 2019 a Montechiaro.

\begin{tabular}{|c|c|c|c|c|c|c|c|c|c|}
\hline \multirow{3}{*}{$\begin{array}{c}\text { Sorte (FAO-Zahl) } \\
\text { Varietà e classe FAO }\end{array}$} & \multirow{3}{*}{$\begin{array}{l}\text { TS-Gehaltw } \\
\text { Sostanza sec- } \\
\text { ca }^{w} \\
(\%)\end{array}$} & \multicolumn{2}{|c|}{ TM-Ertrag } & \multirow{3}{*}{$\begin{array}{l}\text { Stärkegehalt } \\
\text { Amido } \\
\text { (\%) }\end{array}$} & \multirow{3}{*}{$\begin{array}{c}\text { Energiegehalt }{ }^{\mathrm{L}} \\
\text { Concentrazione ener- } \\
\text { geticaL } \\
\text { (MJ NEL/kg TS) }\end{array}$} & \multirow{2}{*}{\multicolumn{2}{|c|}{$\begin{array}{l}\text { Energieertrag } \\
\text { Resa energetica }\end{array}$}} & \multirow{3}{*}{$\begin{array}{l}\text { Fehlstellenw }^{w} \\
\text { Fallanze }^{w} \\
\text { (\%) }\end{array}$} & \multirow{3}{*}{$\begin{array}{c}\text { Nebentriebe }^{W} \\
\text { II e III piantew } \\
\text { (\%) }\end{array}$} \\
\hline & & \multicolumn{2}{|c|}{ Produzione sostanza secca } & & & & & & \\
\hline & & (t/ha) & (rel. \%) & & & (GJ NEL/ha) & (rel. \%) & & \\
\hline Benedictio (230) & $37,5^{a b}$ & 24,6 & 105,8 & 36,5 & $7,92^{\mathrm{a}}$ & 169,8 & 109,3 & $15,5^{\mathrm{cd}}$ & 5,7 \\
\hline Evolino (220) & $35,1^{c}$ & 20,6 & 88,6 & 28,0 & $7,43^{d}$ & 132,8 & 85,5 & $25,0^{\mathrm{a}}$ & 24,5 \\
\hline Figaro (250) & $37,7^{\mathrm{ab}}$ & 24,8 & 106,7 & 33,4 & $7,49 \mathrm{~cd}$ & 161,5 & 104,0 & $17,7^{\mathrm{bcd}}$ & 9,6 \\
\hline Frederico (240) & $37,4^{\mathrm{ab}}$ & 23,8 & 102,4 & 38,7 & $7,70^{a b c}$ & 159,7 & 102,8 & $18,9^{\mathrm{bc}}$ & 11,7 \\
\hline Gottardo (220) & $36,5^{b}$ & 22,4 & 96,4 & 38,6 & $7,78^{\mathrm{ab}}$ & 152,3 & 98,0 & $20,2^{\mathrm{bc}}$ & 7,4 \\
\hline P7515 (220) & $36,6^{b}$ & 22,6 & 97,2 & 38,3 & $7,81^{\mathrm{a}}$ & 154,4 & 99,4 & $17,4^{\mathrm{bcd}}$ & 24,2 \\
\hline P8000 (230) & $38,2^{a}$ & 23,3 & 100,2 & 43,4 & $7,70^{a b c}$ & 156,6 & 100,8 & $12,0^{\text {de }}$ & 20,2 \\
\hline P8201 (240) & $36,4^{\mathrm{bc}}$ & 24,2 & 104,1 & 32,4 & $7,53^{\mathrm{bcd}}$ & 158,3 & 101,9 & $0,4^{e}$ & 12,8 \\
\hline Torres (250) & $37,1^{\mathrm{ab}}$ & 22,9 & 98,5 & 32,5 & $7,67^{\mathrm{abcd}}$ & 152,8 & 98,4 & $20,8^{b}$ & 27,5 \\
\hline $\begin{array}{l}\text { Mittelwert } \\
\text { Media }\end{array}$ & 36,9 & 23,2 & & 35,8 & 7,67 & 155,4 & & 16,4 & 16,0 \\
\hline
\end{tabular}

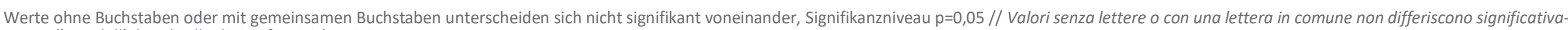
mente l'uno dall'altro, livello di significatività $p=0,05$.

L Auswertung nach logarithmischer Transformation // L Elaborazione dopo trasformazione logaritmica.

W Auswertung nach Winkeltransformation // W Elaborazione dopo trasformazione angolare. 
Tab. 7: Ergebnisse der Silomais-Sortenprüfung 2019 für den Standort Dietenheim // Risultati delle prove varietali 2019 a Teodone.

\begin{tabular}{|c|c|c|c|c|c|c|c|c|c|}
\hline \multirow{3}{*}{$\begin{array}{l}\text { Sorte (FAO-Zahl) } \\
\text { Varietà e classe FAO }\end{array}$} & \multirow{3}{*}{$\begin{array}{l}\text { TS-Gehalt } \\
\text { Sostanza sec- } \\
\mathrm{ca}^{Q} \\
(\%)\end{array}$} & \multirow{2}{*}{\multicolumn{2}{|c|}{$\begin{array}{l}\text { TM-Ertrag } \\
\text { Produzione sostanza secca }\end{array}$}} & \multirow{3}{*}{$\begin{array}{l}\text { Stärkegehalt } \\
\text { Amido } \\
\text { (\%) }\end{array}$} & \multirow{3}{*}{$\begin{array}{l}\text { Energiegehalt }{ }^{\mathrm{L}} \\
\text { Concentrazione ener- } \\
\text { getica }{ }^{\mathrm{L}} \\
\text { (MJ NEL/kg TS) }\end{array}$} & \multirow{2}{*}{\multicolumn{2}{|c|}{$\begin{array}{l}\text { Energieertrag } \\
\text { Resa energetica }\end{array}$}} & \multirow{3}{*}{$\begin{array}{l}\text { Fehlstellen } \\
\text { Fallanze } \\
\text { (\%) }\end{array}$} & \multirow{3}{*}{$\begin{array}{l}\text { Nebentriebew } \\
\text { II e III piantew } \\
\text { (\%) }\end{array}$} \\
\hline & & & & & & & & & \\
\hline & & (t/ha) & (rel. \%) & & & (GJ NEL/ha) & (rel. \%) & & \\
\hline Adevey (280) & $35,0^{\mathrm{bcd}}$ & $25,6^{f}$ & 90,1 & $34,6^{\text {bcdefg }}$ & $6,72^{\text {de }}$ & $172,9^{e}$ & 88,8 & $27,6^{b}$ & $29,4^{\text {def }}$ \\
\hline ATRANS (290) & $31,7^{\mathrm{fg}}$ & $27,7^{\text {bcdef }}$ & 97,5 & $33,1^{\text {defg }}$ & $6,83^{\text {bcde }}$ & $189,8^{\text {cde }}$ & 97,4 & $8,2^{c}$ & $30,0^{\text {de }}$ \\
\hline Figaro (250) & $34,3^{\text {cde }}$ & $30,0^{\mathrm{abcd}}$ & 105,6 & $30,7^{\mathrm{fg}}$ & $6,85^{\text {bcde }}$ & $205,9^{a b c}$ & 105,7 & $4,3^{c}$ & $23,9^{\text {efgh }}$ \\
\hline GIULIANA (280) & $37,0^{a b c}$ & $28,5^{\text {bcdef }}$ & 100,3 & $32,7^{\text {defg }}$ & $6,68^{e}$ & $190,1^{\text {cde }}$ & 97,6 & $0,9^{c}$ & $9,0^{i}$ \\
\hline Hulk (250) & $32,4^{\mathrm{ef}}$ & $26,5^{\text {def }}$ & 93,2 & $37,4^{\mathrm{abcd}}$ & $7,09^{a b}$ & $188,9^{\text {cde }}$ & 97,0 & $3,8^{c}$ & $7,4^{i}$ \\
\hline Kentos (280) & $32,2^{\mathrm{ef}}$ & $30,9^{a b c}$ & 108,7 & $29,3^{g}$ & $6,65^{e}$ & $206,2^{\mathrm{abc}}$ & 105,9 & $3,7^{c}$ & $27,5^{\text {defg }}$ \\
\hline KOKUNA (290) & $35,2^{\mathrm{abcd}}$ & $27,6^{\text {cdef }}$ & 97,1 & $41,7^{a}$ & $7,16^{a}$ & $198,0^{\mathrm{abcd}}$ & 101,7 & $4,2^{c}$ & $32,3^{c d}$ \\
\hline LG30315 (270) & $27,7^{\mathrm{h}}$ & $26,5^{\text {def }}$ & 93,2 & $33,5^{\text {cdefg }}$ & $6,72^{\mathrm{de}}$ & $178,6^{\text {de }}$ & 91,7 & $60,2^{\mathrm{a}}$ & $47,8^{\mathrm{a}}$ \\
\hline MELONGA (270) & $37,2^{\mathrm{ab}}$ & $27,2^{\text {def }}$ & 95,7 & $32,3^{\text {defg }}$ & $6,90^{\text {abcde }}$ & $188,7^{\text {cde }}$ & 96,9 & $9,0^{c}$ & $30,9^{d}$ \\
\hline P7932 (250) & $37,9^{a}$ & $29,5^{\mathrm{abcd}}$ & 103,8 & $36,7^{\mathrm{abcd}}$ & $6,77^{\text {de }}$ & $200,4^{\text {abcd }}$ & 102,9 & $0,2^{c}$ & $22,6^{\mathrm{gh}}$ \\
\hline P8333 (260) & $36,9^{a b c}$ & $28,3^{\text {bcdef }}$ & 99,6 & $34,4^{\text {bcdefg }}$ & $6,78^{\text {cde }}$ & $192,4^{\text {bcde }}$ & 98,8 & $3,3^{c}$ & $23,3^{\text {fgh }}$ \\
\hline P8500 (260) & $34,6^{\text {bcde }}$ & $27,9^{\text {bcdef }}$ & 98,2 & $38,2^{a b c}$ & $6,75^{\text {de }}$ & $187,9^{\text {cde }}$ & 96,5 & $4,3^{c}$ & $16,2^{\mathrm{hi}}$ \\
\hline P8666 (270) & $34,1^{\text {def }}$ & $32,2^{a}$ & 113,3 & $32,3^{\text {defg }}$ & $6,68^{e}$ & $215,3^{\mathrm{ab}}$ & 110,5 & $1,3^{c}$ & $22,2^{\mathrm{gh}}$ \\
\hline P8707 (270) & $34,4^{\text {cde }}$ & $28,9^{\text {abcdef }}$ & 101,7 & $39,7^{a b}$ & $6,72^{\mathrm{de}}$ & $194,6^{\text {bcde }}$ & 99,9 & $1,9^{c}$ & $37,7^{b c}$ \\
\hline P8888 (280) & $32,7^{\text {def }}$ & $28,7^{a b c d e f}$ & 101,0 & $34,6^{\text {bcdef }}$ & $6,92^{\text {abcde }}$ & $199,1^{\mathrm{abcd}}$ & 102,2 & $10,1^{c}$ & $23,8^{\text {fgh }}$ \\
\hline Poesi (280) & $29,4^{\mathrm{gh}}$ & $29,1^{\text {abcde }}$ & 102,4 & $30,8^{\text {efg }}$ & $6,86^{\text {bcde }}$ & $199,4^{\mathrm{abcd}}$ & 102,4 & $6,1^{c}$ & $11,6^{i}$ \\
\hline
\end{tabular}




\begin{tabular}{|c|c|c|c|c|c|c|c|c|c|}
\hline Torres (250) & $34,7^{\text {bcde }}$ & $25,6^{\mathrm{ef}}$ & 90,1 & $36,1^{\text {abcde }}$ & $6,99^{a b c d}$ & $179,3^{\text {de }}$ & 92,1 & $21,6^{b}$ & $39,5^{b}$ \\
\hline Walterinio (270) & $33,0^{\text {def }}$ & $30,9^{\mathrm{ab}}$ & 108,7 & $39,2^{\mathrm{ab}}$ & $7,06^{a b c}$ & $218,5^{a}$ & 112,2 & $4,3^{c}$ & $7,1^{i}$ \\
\hline $\begin{array}{l}\text { Mittelwert } \\
\text { Media }\end{array}$ & 33,9 & 28,4 & & 34,9 & 6,84 & 194,8 & & 9,7 & 24,6 \\
\hline
\end{tabular}

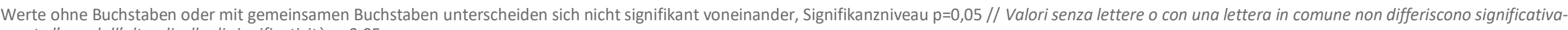
mente l'uno dall'altro, livello di significatività $p=0,05$.

Q Auswertung nach Quadratwurzeltransformation // Q Elaborazione dopo trasformazione con radice quadrata.

W Auswertung nach Winkeltransformation // W Elaborazione dopo trasformazione angolare. 
Tab. 8: Ergebnisse der Silomais-Sortenprüfung 2019 für den Standort Gufidaun // Risultati delle prove varietali 2019 a Gudon.

\begin{tabular}{|c|c|c|c|c|c|c|c|c|c|}
\hline \multirow{3}{*}{$\begin{array}{l}\text { Sorte (FAO-Zahl) } \\
\text { Varietà e classe } \\
\text { FAO }\end{array}$} & \multirow{3}{*}{$\begin{array}{l}\text { TS-Gehalt } \\
\text { Sostanza } \\
\text { secca } \\
\text { (\%) }\end{array}$} & \multirow{2}{*}{\multicolumn{2}{|c|}{$\begin{array}{c}\text { TM-Ertrag } \\
\text { Produzione sostanza secca }\end{array}$}} & \multirow{3}{*}{$\begin{array}{l}\text { Stärkegehalt } \\
\text { Amido } \\
\text { (\%) }\end{array}$} & \multirow{3}{*}{$\begin{array}{c}\text { Energiegehalt }{ }^{\mathrm{L}} \\
\begin{array}{c}\text { Concentrazione ener- } \\
\text { getical }\end{array} \\
\text { (MJ NEL/kg TS) }\end{array}$} & \multirow{2}{*}{\multicolumn{2}{|c|}{$\begin{array}{l}\text { Energieertrag } \\
\text { Resa energetica }\end{array}$}} & \multirow{3}{*}{$\begin{array}{c}\text { Fehlstellen } \\
\text { Fallanze } \\
\text { (\%) }\end{array}$} & \multirow{3}{*}{$\begin{array}{c}\text { Nebentriebe }{ }^{W} \\
\text { II e III piantew } \\
\text { (\%) }\end{array}$} \\
\hline & & & & & & & & & \\
\hline & & (t/ha) & (rel. \%) & & & (GJ NEL/ha) & (rel. \%) & & \\
\hline FREEMAN (400) & $32,2^{b}$ & 30,1 & 101,7 & 36,1 & 7,53 & 197,6 & 101,6 & 6,8 & $30,0^{\text {def }}$ \\
\hline P0362 (400) & $30,9 \mathrm{bc}$ & 29,1 & 98,3 & 40,1 & 7,70 & 195,3 & 100,4 & 4,9 & $25,9^{\mathrm{fg}}$ \\
\hline P0729 (400) & $33,3^{b}$ & 31,8 & 107,4 & 34,3 & 7,63 & 210,7 & 108,3 & 3,3 & $39,3^{b c}$ \\
\hline P0837 (400) & $31,5^{b}$ & 31,6 & 106,8 & 36,6 & 7,67 & 211,6 & 108,7 & 4,7 & $44,7^{a b}$ \\
\hline P1275 (500) & $27,3^{\text {cd }}$ & 29,1 & 98,3 & 31,8 & 7,49 & 189,4 & 97,3 & 6,5 & $20,1^{\mathrm{fg}}$ \\
\hline P9911 (300) & $33,3^{b}$ & 32,3 & 109,1 & 36,1 & 7,53 & 211,6 & 108,7 & 4,6 & $24,6^{\mathrm{fg}}$ \\
\hline SIV4795 (300) & $34,4^{\mathrm{ab}}$ & 26,2 & 88,5 & 39,7 & 7,56 & 171,8 & 88,3 & 4,7 & $35,8^{\text {cde }}$ \\
\hline SIV5031 (300) & $31,5^{b}$ & 27,1 & 91,6 & 38,4 & 7,49 & 177,1 & 91,0 & 2,2 & $37,7^{b c d}$ \\
\hline SIV6181 (500) & $27,2^{d}$ & 29,4 & 99,3 & 33,5 & 7,53 & 192,6 & 99,0 & 7,2 & $26,6^{\mathrm{efg}}$ \\
\hline SY Carioca (400) & $32,3^{b}$ & 27,9 & 94,3 & 37,3 & 7,46 & 181,0 & 93,0 & 3,0 & $24,9^{\mathrm{fg}}$ \\
\hline SY Senko (400) & $31,9^{b}$ & 28,8 & 97,3 & 37,0 & 7,48 & 187,3 & 96,3 & 3,6 & $18,8^{g}$ \\
\hline SY Zephir (300) & $37,1^{\mathrm{a}}$ & 31,8 & 107,4 & 37,3 & 7,56 & 208,9 & 107,4 & 6,0 & $47,4^{a}$ \\
\hline $\begin{array}{l}\text { Mittelwert } \\
\text { Media }\end{array}$ & 31,9 & 29,6 & & 36,5 & 7,55 & 194,6 & & 4,8 & 31,3 \\
\hline
\end{tabular}

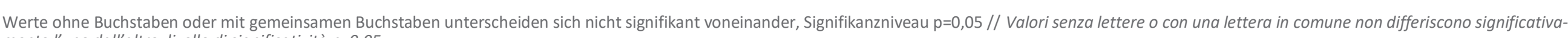
mente l'uno dall'altro, livello di significatività $p=0,05$.

L Auswertung nach logarithmischer Transformation // L Elaborazione dopo trasformazione logaritmica.

W Auswertung nach Winkeltransformation // W Elaborazione dopo trasformazione angolare. 


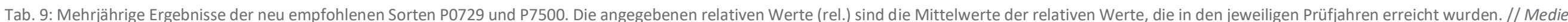
pluriennali delle nuove varietà inserite nella lista di consulenza varietale. I valori relativi (rel.) sono la media dei valori relativi in percentuale riferiti alla media della varietà testate nello stesso anno.

\begin{tabular}{|c|c|c|c|c|c|c|c|c|c|c|c|}
\hline Sorte (FAO-Zahl) & Firma & Anzahl Prüfjahre & TS-Gehalt & \multicolumn{2}{|c|}{ TM-Ertrag } & \multicolumn{2}{|c|}{ Stärkegehalt } & \multicolumn{2}{|c|}{ Energiegehalt } & \multicolumn{2}{|c|}{ Energieertrag } \\
\hline \multirow{2}{*}{$\begin{array}{c}\text { Varietà (numero } \\
\text { FAO) }\end{array}$} & Ditta & Numero di anni di & Sostanza & Resa in & nza secca & & & Contenuto & etico & Resa ene & ica \\
\hline & & & $(\%)$ & (t/ha) & (rel. \%) & (abs. \%) & (rel. \%) & (MJ NEL/kg) & (rel. \%) & (GJ NEL/ha) & (rel. \%) \\
\hline P0729 (400) & Pioneer & 3 & 32,9 & 31,6 & 107,2 & 35,4 & 102,7 & 6,92 & 101,4 & 208,6 & 109,2 \\
\hline P7500 (210) & Pioneer & 5 & 32,7 & 21,5 & 99,5 & 34,0 & 101,8 & 6,93 & 101,0 & 149,0 & 100,7 \\
\hline
\end{tabular}




\section{ANHANG 2: ABBILDUNGEN}
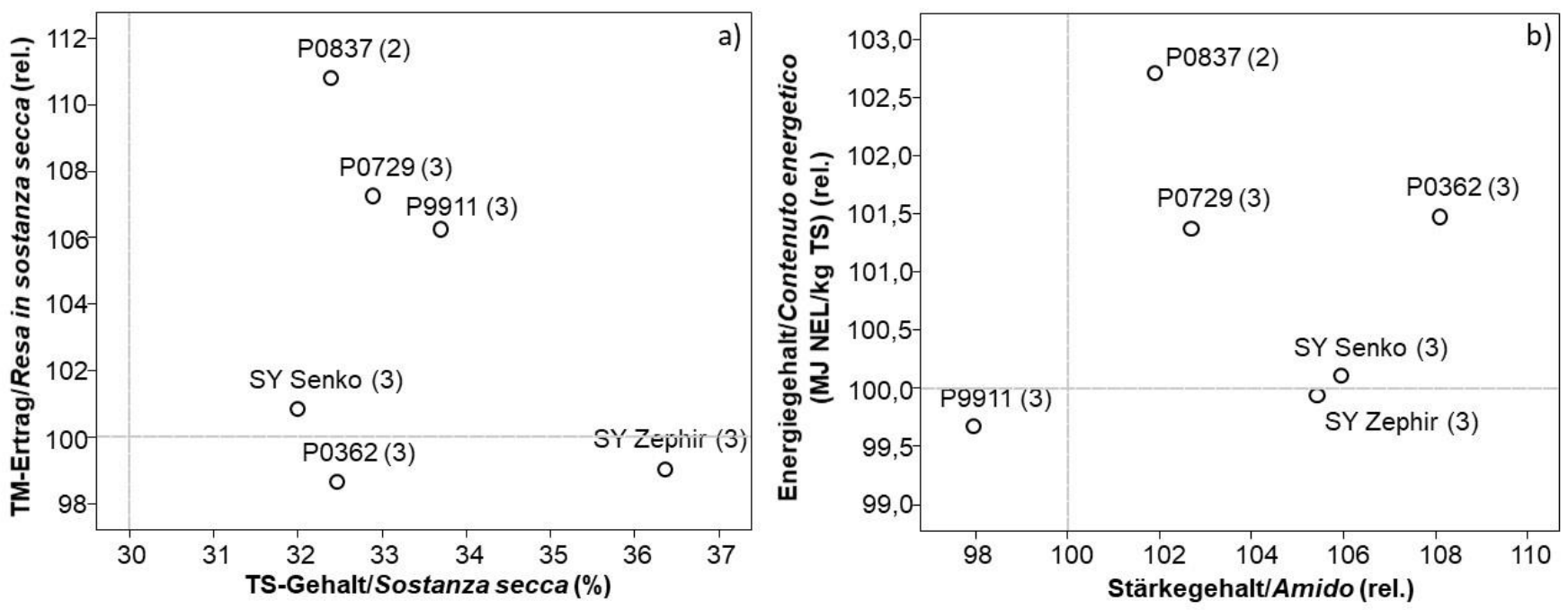

Abb. 1: Gegenüberstellung von TS-Gehalt und TM-Ertrag (a) sowie von Stärkegehalt und Energiegehalt (b) von im Jahr 2019 schon mindestens zweimal geprüften Sorten am Standort Gufidaun. Die relativen Werte beziehen sich auf den Mittelwert des Sortiments im jeweiligen Prüfjahr. Die Zahlen in Klammern stehen für die Anzahl an Prüfjahren. // Confronto tra la sostanza secca e la resa in sostanza secca (a) e tra amido e contenuto energetico (b) delle varietà testate nel 2019 da almeno due anni nel sito di Gudon. I valori relativi si riferiscono al valore medio di tutte le varietà testate nel rispettivo anno di prova. I numeri tra parentesi rappresentano il numero di anni di prova. 

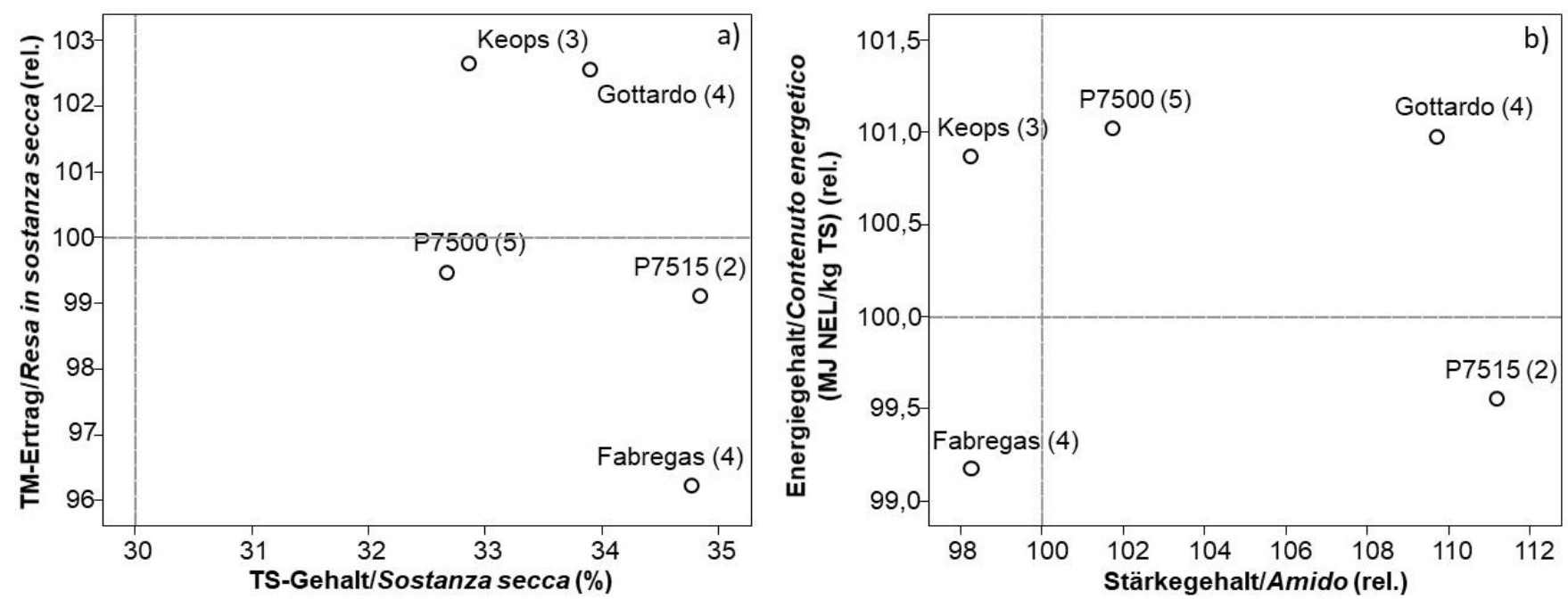

Abb. 2: Gegenüberstellung von TS-Gehalt und TM-Ertrag (a) sowie von Stärkegehalt und Energiegehalt (b) von im Jahr 2019 schon mindestens zweimal geprüften Sorten am Standort Olang. Die relativen Werte beziehen sich auf den Mittelwert des Sortiments im jeweiligen Prüfjahr. Die Zahlen in Klammern stehen für die Anzahl an Prüfjahren. // Confronto tra la sostanza secca e la resa in sostanza secca (a) e tra amido e contenuto energetico (b) delle varietà testate nel 2019 da almeno due anni nel sito di Valdaora. I valori relativi si riferiscono al valore medio di tutte le varietà testate nel rispettivo anno di prova. I numeri tra parentesi rappresentano il numero di anni di prova.

\section{(c) (1) (8)}

Dieses Werk ist lizenziert unter einer Creative Commons Namensnennung-Nicht kommerziell 4.0 International Lizenz. Quest'opera è distribuita con Licenza Creative Commons Attribuzione - Non commerciale 4.0 Internazionale. This work is licensed under a Creative Commons Attribution-NonCommercial 4.0 International License.

Für alle Abbildungen und Tabellen ohne Nennung des Urhebers gilt: @ Versuchszentrum Laimburg. Per tutte le immagini e tabelle senza menzione dell'artefice vale: ( Centro di Sperimentazione Laimburg. For all figures and tables without mention of the originator applies: () Laimburg Research Centre. 